\title{
Tuning Molecular Recognition of RNA and DNA via Sugar Modification
}

\author{
Christian J. Leumann*
}

\begin{abstract}
This minireview highlights three aspects of our recent work in the area of sugar modified oligonucleotide analogues. It provides an overview over recent results on the conformationally constrained analogue tricyclo-DNA with special emphasis of its antisense properties, it summarizes results on triple-helix forming oligodeoxynucleotides containing pyrrolidino-nucleosides with respect to DNA recognition via the dual recognition mode, and it highlights the advantageous application of the orthogonal oligonucleotidic pairing system homo-DNA in molecular beacons for DNA diagnostics.
\end{abstract}

Keywords: Antisense - DNA recognition - DNA triple-helix · Molecular beacons · Pyrrolidino nucleosides

\section{Introduction}

Oligonucleotides have found widespread interest and application in DNA and RNA diagnostics, as tools in molecular biology and as antisense agents and gene silencers in functional genomics and human therapy. For many of these applications, however, unmodified oligonucleotides are of limited use due to their insufficient biostability, bioavailability and due to the fact that their affinity to complementary DNA and RNA is in many instances not sufficient to generate a biological response. Some of these drawbacks can be overcome by chemical modification of oligonucleotides [1]. All of the three components of the repetitive structural unit of DNA or RNA, namely the sug-

${ }^{*}$ Correspondence: Prof. Dr. C.J. Leumann Department of Chemistry and Biochemistry University of Bern

Freiestrasse 3

$\mathrm{CH}-3012$ Bern

Tel.: +41316314355

Fax: +41316313422

E-Mail: leumann@ioc.unibe.ch ar, the phosphate and the nucleobase, are equally amenable to chemical modification to improve on desired properties. Modifications at the sugar unit, ranging from simple substitutions at the 2 -hydroxyl group to complete replacement of the sugar by different structural scaffolds have the most profound effect on the pairing properties of derived oligonucleotides. For example, changing the geometry of some of the torsion angles in the backbone, as for example in the case of homo-DNA, bc-DNA or xyloDNA leads to base association preferences different from that of the Watson-Crick type, or to orthogonal base-pairing systems. On the other side, conformational constriction or preorganization of backbone torsion angles in the sugar unit as in the case of LNA or tricyclo-DNA (tc-DNA) leads to increased complex stability by maintaining the typical Watson-Crick association mode. Therefore understanding the relation between sugar structure and pairing properties in sufficient detail is the key for tuning oligonucleotide properties at will by chemical modification.

In the following we concentrate on three different sugar modifications that we have recently investigated in three different contexts. First we summarize recent results with conformationally constrained oligonucleotides showing increased RNA affinity, then we focus on a combination of specific base and unspecific phosphate recognition as a means to increase triple-helix stability, and in the last section we give an example on how orthogonal oligonucleotidic basepairing systems that do not cross-pair with natural nucleic acids, can advantageously be used in biotechnology.

\section{Conformationally Constrained Oligonucleotides for RNA Recognition}

Over the last years we developed the DNA analogue tricyclo-(tc)DNA (Fig. 1). As LNA, tc-DNA belongs to the class of conformationally constrained oligonucleotide analogues. These were specifically designed to increase complementary RNA or DNA affinity by reducing the entropy change upon duplex formation via structural preorganization of the single strand. We reported in the past on the synthesis and duplex formation properties of tricyclo-DNA and found indeed reduced entropy changes upon duplex formation and increased thermostability of duplexes with RNA by $2-4$ ${ }^{\circ} \mathrm{C}$ in melting temperature per modification relative to DNA [2][3]. With this it is one of the strongest RNA-binders. Structurally tc-DNA was found to be an RNA mimic as deduced from CD-spectroscopic and modeling experiments. Furthermore, tc-DNA is very stable against 3 '-phosphodiesterases (snake venom phosphodiesterase) and is

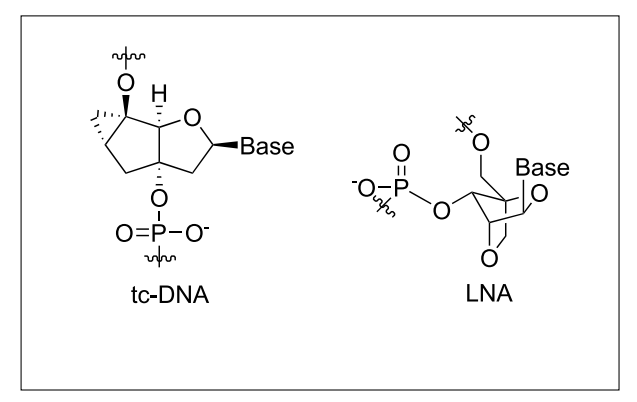

Fig. 1. Chemical structures of tricyclo-DNA and LNA 
also stable in sera and cell extracts. In addition we found that tcDNA/RNA hybrid duplexes do not elicit RNase $\mathrm{H}$ activity. These properties clearly prompted us to perform nuclear antisense experiments on two different targets. During these experiments we also compared the properties of tc-DNA to those of commercially available and widely used 2'-OMe-RNA and locked nucleic acid (LNA).

We tested for antisense effects in HeLa cell lines stably expressing the human $\beta$ globin gene with two different point mutations in the second intron. These mutations lead to the inclusion of an aberrant exon in $\beta$-globin mRNA. Lipofectamine-mediated delivery of a 17-mer tricyclo-oligodeoxynucleotide complementary to the cryptic $3^{\prime}$ splice site at the beginning of the aberrant exon results in correction of splicing already at $\mathrm{nM}$ concentrations with up to 100 -fold enhanced efficiency relative to a 2 '-OMe-PS-RNA oligonucleotide of the same length and sequence. In contrast to 2'-OMe-PS-RNA, tricyclo-DNA shows antisense activity even in the absence of lipofectamine, albeit only at much higher oligonucleotide concentrations [4].

In another setup the nuclear antisense properties of a series of tricyclo(tc)-DNA oligonucleotide 9-15mers, targeted against the $3^{\prime}$ and $5^{\prime}$ splice sites of exon 4 of cyclophilin A (CyPA) pre-mRNA, were evaluated in HeLa cells and compared with those of corresponding LNA-oligonucleotides [5]. While the 9mers showed no significant antisense effect, the 11-15mers induced single and double exon skipping to about an equal extent upon lipofectamine mediated transfection in a sequence and dose dependent manner, as revealed by a RT-PCR assay. The antisense efficacy of the tc-oligonucleotides was found to be superior to that of the LNA-oligonucleotides in all cases by a factor of at least 4-5. A tc-oligonucleotide $15 \mathrm{mer}$ completely abolished CyPA mRNA production at $0.2 \mu \mathrm{M}$ concentration. The antisense effect was confirmed by western blot analysis which revealed a reduction of CyPA protein to $13 \%$ of its normal level. Fluorescence microscopic investigations with a fluorescein labeled tc-15mer revealed a strong propensity for homogeneous nuclear localization of this backbone type after lipofectamine mediated transfection, while the corresponding LNA 15 mer showed a less clear cellular distribution pattern. Transfection without lipid carrier showed no significant internalization of both tc- and LNA-oligonucleotides. The comparison of LNA with tcDNA in the latter experiment surprisingly revealed that there is no linear correlation between RNA affinity and antisense efficacy in this case, indicating that other factors such as intracellular distribution may critically determine the efficacy of oligonucleotides. The current state of evaluation of tcDNA clearly shows that it is an excellent candidate for nuclear antisense applications.

RNase $\mathrm{H}$ activity leading to the degradation of the RNA is an important antisense mechanism that increases the efficacy of antisense oligonucleotides when targeted to mature mRNAs. A way out of the dilemma that many chemically modified oligonucleotide analogues, including tcDNA, are unable to activate RNase $\mathrm{H}$ consists in the use of so-called 'gapmers', chimaeric oligonucleotides in which both ends consist of chemically modified units while the center of the sequence contains 6-10 unmodified DNA-residues (RNase $\mathrm{H}$ window). We therefore decided to explore whether the gapmer concept can also be applied to tc-DNA [6]. A 18mer tc-DNA gapmer containing a DNA window of eight nucleotides in the center, flanked by five tc-DNA residues each on the $5^{\prime}$ - and the $3^{\prime}$-side was found to be as competent as normal DNA in eliciting $\mathrm{RNase} \mathrm{H}$ activity. This gapmer was also largely resistant to biodegradation in both human and fetal calf serum. Thus all the advantages of the gapmer strategy originally developed for 2'-O-alkyl RNA also apply to the tc-DNA scaffold.

\section{Concomittant Base and Phosphate Recognition for Improving DNA Triple Helix Stability}

Sequence specific binding of triplex forming oligonucleotides (TFOs) in the major groove of double stranded DNA can control and modulate gene expression at the level of transcription and is the basis of antigene technology [7]. TFOs can bind to homopurine/homopyrimidine tracts of DNA in either parallel or antiparallel alignment relative to the purine target strand, forming Hoogsteen or reversed-Hoogsteen basetriples. Unfortunately, the use of TFOs as antigene agents suffers from several limitations. Low thermal stability of the triplexes, low biostability of the TFOs in vivo, low bioavailability, sequence restrictions to homopurine/homopyrimidine DNA tracts and a strong $\mathrm{pH}$ dependence in the parallel binding motif due to $\mathrm{C}^{+} \bullet \mathrm{G}-\mathrm{C}$ triplet formation are among the major obstacles that reduce the efficacy of antigene agents and that slow down the development and applications of antigene technology. Many strategies have been exploited to avoid these drawbacks, including the use of conformationally restricted DNA analogues to increase thermal stability [8], or the design of new selective nucleobase analogues to alleviate target sequence restriction [9][10].

As part of our current research we are interested in increasing TFO affinity to a DNA target by applying the 'dual recognition' approach, originally developed by
Cuenoud and coworkers [11]. In this approach, a target dsDNA is not only recognized by selective base-base interactions but also via an additional, non-specific salt bridge between an appropriately placed positive charge in the TFO and a negatively charged phosphate oxygen in the target DNA backbone [11][12].

Based on recent X-ray data on a parallel DNA triplex [13], molecular modeling showed that replacement of the 4 '-oxygen in a deoxynucleoside residue of a TFO by a basic nitrogen would potentially place a positive charge next to a non-bridging pro$R$-phosphate oxygen of the purine strand of a target dsDNA (Fig. 2). We therefore prepared the corresponding pyrrolidinopseudonucleosides and incorporated them into oligonucleotides. TFOs containing pyrrolidino pseudoisocytidine units (dp $\psi \mathrm{iC}$-series) exhibit a significant increase in triplex stability compared to unmodified TFOs in at least two different sequence contexts [14]. By comparison with TFOs containing ribo-pseudo-isoC units (druiC-series) it clearly emerges that the increase in stability is associated with the protonation of the pyrrolidino ring nitrogen which confirms the presence of attractive electrostatic interactions between the additional positive charge in the TFOs and the phosphodiester backbone of the target duplex. A somewhat puzzling situation remains in that this additional electrostatic stabilization seems to be restricted to dp $\psi \mathrm{iC}$ units and does not occur with $d p \psi \mathrm{U}$ units for a yet unknown reason.

\section{Orthogonal Base-pairing Systems for Applications in DNA Diagnostics}

The post-genomic era continuously demands novel and improved DNA recognition probes with high sensitivity and selectivity for use in fundamental biomedical studies, disease diagnosis, functional genomics and drug discovery. A particularly useful concept for sensing single-stranded DNA or RNA with high mismatch discrimination is that of the molecular beacons (MBs), first described by Kramer and coworkers [15]. MBs are single-stranded oligonucleotide hybridization probes that form a stem-loop structure. The loop contains a probe sequence that is designed to be complementary to a DNA-target, and the stem is formed by complementary arm sequences that are located on either side of the probe. A fluorophore is covalently linked to the end of one arm and a quencher to the end of the other arm. In the absence of a target the molecular beacon is closed, and fluorescence is quenched. However, after hybridization to a nucleic acid target sequence the stem of the molecular beacon melts and fluorescence is emitted due to in- 

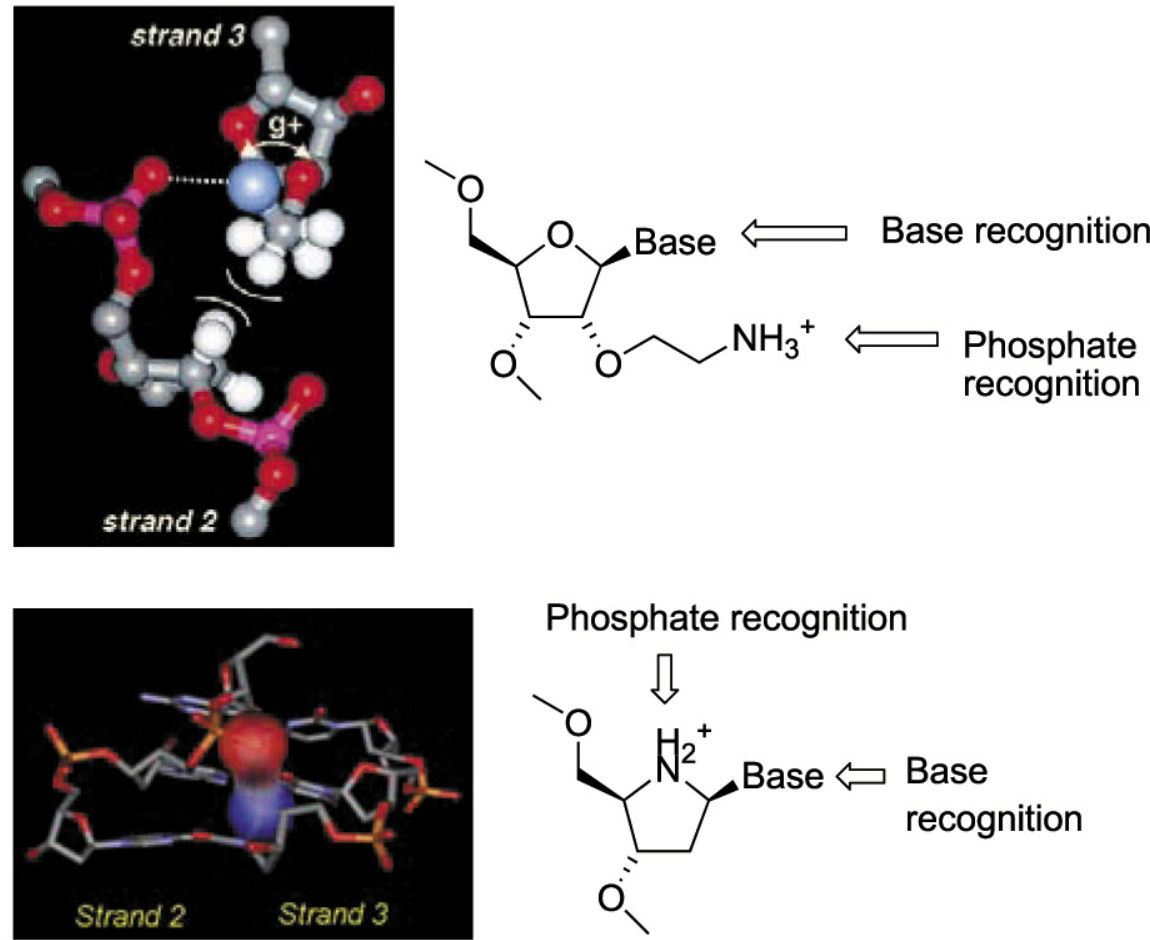

Phosphate recognition<smiles>[X]C1CCC(c2cn([R])c(=O)[nH]c2=O)C1</smiles>

$\mathrm{HO}$

$$
\begin{aligned}
& \operatorname{dp} \psi \mathrm{U}, \mathrm{X}=\mathrm{NH}_{2}, \mathrm{R}=\mathrm{H} \\
& \operatorname{dp} \psi \mathrm{T}, \mathrm{X}=\mathrm{NH}_{2}, \mathrm{R}=\mathrm{CH}_{3} \\
& \operatorname{dr} \psi \mathrm{U}, \mathrm{X}=\mathrm{O}, \mathrm{R}=\mathrm{H}
\end{aligned}
$$

creased distance between fluorophore and quencher (Fig. 3A).

MB probes display higher target selectivity compared to linear probes, due to their structurally well-defined hairpin loop state in the absence of a target [16]. The MB concept has been developed and exploited extensively in the past for the detection of single nucleotide polymorphisms [17], for visualizing the distribution and transport of mRNAs in living cells [18], as probes for reporting enzymatic activity [19], as well as for a variety of other applications [20]. Although molecular beacons are very sensitive to mismatches in the probe-target region their flanking arm sequences, containing 4-5 nucleotides each are prone to fortuitously participate in target binding, thus constituting a potential source of false positive results. A solution to this problem exists in theory by replacing the stem part of an MB by an oligonucleotidic pairing system that is orthogonal and consequently does not cross-pair with DNA or RNA.

In our approach we chose homo-DNA (Fig. 3B) for the stem part of MBs. HomoDNA, a homologue of natural DNA, was shown before by Eschenmoser and coworkers to form stable, antiparallel duplexes with itself, without cross-pairing to natural DNA [21]. Moreover, homo-DNA can easily be prepared by standard automated phosphoramidite chemistry and is thus fully compatible with DNA synthesis [22]. Particularly intriguing is the property of homo-DNA oligoadenylates to form A-A self-pairs of the reversed Hoogsteen type (Fig. 3C) with thermal stabilities that roughly match that of natural Watson-Crick base-pairs. Exploiting this property reduces the number of necessary building blocks for the arms of an MB to only one.

We found that hybrid MBs with a homoadenylate homo-DNA stem and a DNA probe sequence (Fig. 3D) readily form hairpin-loop structures with a stability that roughly matches that of an unmodified MB [23]. No interaction between the homoDNA sequence tracts with the target DNA was observed. By targeting with the loop region a single sequence in a DNA array we could show that such hybrid homoDNA beacons significantly enhanced target selectivity compared to conventional beacons. The signal to background ratios of the homo-DNA molecular beacons were found to be similar to that of the conventional MBs as deduced from fluorescence melting profiles of the beacons alone as well as with target.

The use of non-natural oligonucleotidic pairing systems in biotechnology is appealing and only little explored. Among the few examples are L-DNA that has been used in the past as aptamers (spiegelmers) for selective binding of biopolymers [24], pyranosyl RNA (pRNA) as a nanosystem

Fig. 3. A: Working principle of the molecular beacon technology; B: Chemical structure of homo-DNA; C: Reversed Hoogsteen A-A base-pairing as found in homo-DNA homoadenylates; D: Schematic representation of homo-DNA/DNA hybrid molecular beacons 
[25], and the 3'-deoxy variant of pRNA (pDNA) as a scaffold for tuning aptamer and ribozyme activity [26]. The molecular beacon approach described above is the first application of homo-DNA in nucleic acid biotechnology.

\section{Conclusions}

Oligonucleotide chemistry has already provided many tools for molecular biology and has been instrumental for the success of functional genomics. As many other fields in science, also DNA chemistry has experienced various cycles of low and high tides since the development of solid phase DNA synthesis. Waves of interest were first associated with site-directed mutagenesis and PCR, then with antisense research as well as selection/amplification protocols for the generation of ribozymes and aptamers, and most recently with small interfering RNAs and micro RNAs. Although many aspects of the chemistry of this molecule have already been explored, there is still plenty of room for developing novel and improved tools to meet tomorrow's needs in DNA diagnosis and in oligonucleotide dependent therapies.

Received: September 20, 2005
[1] J. Kurreck, Eur. J. Biochem. 2003, 270, 1628-1644.

[2] R. Steffens, C.J. Leumann, J. Am. Chem. Soc. 1999, 121, 3249-3255.

[3] D. Renneberg, C.J. Leumann, J. Am. Chem. Soc. 2002, 124, 5993-6002.

[4] D. Renneberg, D. Schümperli, C.J. Leumann, Nucleic Acids Res. 2002, 30, 27512757.

[5] D. Ittig, S. Liu, D. Renneberg, D. Schümperli, C.J. Leumann, Nucleic Acids Res. 2004, 32, 346-353.

[6] D. Ittig, D. Renneberg, D. Vonlanthen, S. Luisier, C.J. Leumann, Coll. Symp. Series, Vol. 7, Ed. M. Hocek, Prague, 2005, 21-26.

[7] S. Buchini, C.J. Leumann, Curr. Opin. Chem. Biol. 2003, 7, 717-726.

[8] S. Obika, T. Uneda, T. Sugimoto, D. Nanbu, T. Minami, T. Doi, T. Imanishi, Bioorg. Med. Chem. 2001, 9, 1001-1011.

[9] S. Obika, Y. Hari, M. Sekiguchi, T. Imanishi, Chem. Eur. J. 2002, 8, 4796-4802.

[10] I. Prévot-Halter, C. Leumann, Bioorg. Med. Chem. Lett. 1999, 9, 2657-2660.

[11] B. Cuenoud, F. Casset, D. Hüsken, F. Natt, R.M. Wolf, K.-H. Altmann, P. Martin, H.E. Moser, Angew. Chem. Int. Ed. 1998, 37, 1288-1291.

[12] M.J.J. Blommers, F. Natt, W. Jahnke, B. Cuenoud, Biochemistry 1998, 37, 17714 17725.

[13] S. Rhee, Z.-J. Han, K. Liu, H.T. Miles, D.R. Davies, Biochemistry 1999, 38, 1681016815.
[14] A. Mayer, A. Häberli, C.J. Leumann, Org. Biomol. Chem. 2005, 3, 1653-1658.

[15] S. Tyagi, F.R. Kramer, Nat. Biotechnol. 1996, 14, 303-308.

[16] G. Bonnet, S. Tyagi, A. Libchaber, F.R. Kramer, Proc. Natl. Acad. Sci. USA 1999, 96 , 6171-6176.

[17] S. Tyagi, D.P. Bratu, F.R. Kramer, Nat. Biotechnol. 1998, 16, 49-53.

[18] D.P. Bratu, B.J. Cha, M.M. Mhlanga, F.R. Kramer, S. Tyagi, Proc. Natl. Acad. Sci. USA 2003, 100, 13308-13313.

[19] L.M. Kundu, L.T. Burgdorf, O. Kleiner, A. Batschauer, T. Carell, ChemBioChem. 2002 3, 1053-1060.

[20] W. Tan, K. Wang, T.J. Drake, Curr. Opin. Chem. Biol. 2004, 8, 547-553.

[21] J. Hunziker, H.-J. Roth, M. Böhringer, A. Giger, U. Diederichsen, M. Göbel, R. Krishnan, B. Jaun, C. Leumann, A. Eschenmoser, Helv. Chim. Acta 1993, 76, 259-352.

[22] M. Böhringer, H.-J. Roth, J. Hunziker, M. Göbel, R. Krishnan, A. Giger, B. Schweizer, J. Schreiber, C. Leumann, A. Eschenmoser, Helv. Chim. Acta 1992, 75, 1416-1477.

[23] C. Crey-Desbiolles, D.R. Ahn, C.J. Leumann, Nucleic Acids Res. 2005, 33, e77.

[24] D. Eulberg, K. Buchner, C. Maasch, S. Klussmann, Nucleic Acids Res. 2005, 33 , e45.

[25] A. Eschenmoser, C. Miculka, N. Windhab, (Hoechst AG) EP 06907, 1998.

[26] D. Ackermann, X. Wu, S. Pitsch, Helv. Chim. Acta 2002, 85, 1463-1478. 